\title{
Normal Cumulative Distribution Function and Dispersion Entropy Based EMG Classification
}

\author{
iD Muzaffer Aslan ${ }^{1}$ \\ ${ }^{1}$ Corresponding Author; Bingol University Electric \& Electronics Engineering Departmant; \\ muzafferaslan@bingol.edu.tr;+904262160012/1938
}

Received 16 October 2020; Revised 25 November 2020; Accepted 10 December 2020; Published online 30 December 2020

\begin{abstract}
Electromyography (EMG) is used to measure muscle activity. EMG signals are widely used in many biomedical practices such as motion recognition, prosthetic control, physical rehabilitation, and human-computer interfaces. The effective use of EMG in such practices depends on distinctive feature extraction. In this study, Dispersion Entropy (DisEn) and Normal Cumulative Distribution Function (NCDF) methods are used for feature extraction from EMG signals. The suggested method was tested with a data set containing immersion of six different objects. In the experimental studies, the proposed method distinguished the movements with an accuracy performance of $98 \%$. When compared to other methods using the same data set, the suggested method has about $1.2 \%$ better performance.
\end{abstract}

Keywords: EMG, normal cumulative distribution function, dispersion entropy, SVM, ELM

\section{Introduction}

In vertebrates, the contraction of skeletal muscles connected to the bone ensures the movement in parts of the living creature. During the movement contraction of skeletal muscles generates trical signals called electromyography (EMG). These signals are generated due to the change in electrical potential as a result of chemical changes composed by the contraction of neurons in multiple fibers in muscles, called myofibrils. EMG signals contain very important information about muscle activities. Therefore, it is generally used as an input signal at interfaces of human-myoelectric control systems. Today, EMG signals are very popular to use in many areas such as hand gesture recognition, robotics, prosthesis control, video games, and sign language recognition [1]. Therefore, EMG classification is being studied extensively in the literature. Jonior et al. [1] high dimensional EMG data features the size of EMG features are reduced through dimension reduction methods such as Principal Component Analysis (PCA), Linear Discriminant Analysis (LDA), automatic encoder, t-distributed stochastic neighbor embedding and Large Margin Nearest Neighbor (LMMN) reduced. Then, these features were classified with an average accuracy of $89.4 \%$ with the Extreme Learning Machine (ELM) and 94\% with the Support Vector Machine (SVM). Kumari et al. [2] classified the time domain and time scale properties of EMG signals with SVM to facilitate the life of partly disabled people. In their study, feature extraction was performed with Wavelet Packet Transform (WPT) method using 303 EMG data obtained from 8 participants between the ages of 18-30. In experimental studies, they calculated with an accuracy rate of $92.1 \%$ in prosthesis lengthening and $90.5 \%$ accuracy in flexion (bending the joint) operation. They also achieved $91.1 \%$ classification accuracy for elbow movement that can be used to control any bidirectional robotic tool. For the classification of physical actions, Sravani et al. [3] made a classification with ELM after performing feature extraction by dividing into eight sub-bands namely; Flexible analytical wavelet transform, negative entropy, Mean Absolute Value (MAV), variance, modified Mean Absolute Value type 1 (MAV1), waveform length, simple square integral and Tsallis entropy. They achieved the highest performance in all sub-bands and an average accuracy rate of $99.36 \%$ on the seventh sub-band. Arl et al. [4] made use of time frequency representations, Local Binary Pattern (LBP) of each window by segmenting and gray level co-occurrence matrix methods in order to distinguish 6 different actions from EMG signals for feature extraction. The extracted features were classified with Artificial Neural Network (ANN), providing nearly 92\% success. Sapsanis [5], used ELM method to distinguish six basic actions of the hand after eight different feature extraction from 
raw EMG data by decomposing the Surface EMG (sEMG) data set obtained using two electrodes connected to two specific muscles of the hand into Intrinsic Mode Functions (IMFs) using Empirical Mode Decomposition (EMD). They classified the actions with an average accuracy rate of $89.2 \%$ in their study. Qi et al.[6], used LDA and ELM methods to reduce unnecessary information in sEMG signals in hand gestures recognition and to increase recognition efficiency and accuracy. In the study, firstly, features were extracted from EMG signals by root mean square, waveform length, and median amplitude spectrum methods. Then, these high dimensional features were reduced by using LDA and applied to the ELM classifier. They also established an accuracy rate of $79.32 \%$ by optimizing the initial conditions and thresholds of the network with the genetic algorithm to increase the ELM performance. Chaya et al. [7] tried to recognize the movement of the arm with sEMG signals received from the elbow and wrist parts of the arm with a real-time application. After extracting features from EMG signals with methods such as Average Absolute Value (AAV), variance, standard deviation, Root Mean Square (RMS), Power Spectral Entropy (PSE), average frequency, they achieved 93.3\% success with SVM in the classification of both elbow and wrist positions. Ar1 et al. [8] conducted feature extraction using Permutational Entropy (PE) and LBP methods to classify 6 different hand gestures. The features were classified with SVM after being normalized with zero-unit variance. In the experimental studies, an average of $93.1 \%$ performance was achieved in six hand movements. Tuncer et al. [9] utilized k-Nearest Neighbor (k-NN) methods for triple pattern, Discrete Wavelet (DWT) based feature extraction and classification using EMG signals for the control of prosthetic hands of amputated people. In experimental studies using an EMG data set with 3 strength levels (Low, Moderate, High) collected from amputated volunteers, hand movements for low, moderate, and high strength levels were determined with accuracy rates of $97.78 \%, 93.33 \%$, and $92.96 \%$, respectively. They also achieved a classification performance of $99.14 \%$ for all strength levels. Turlapaty et al. [10] features obtained from different methods such as interchannel time statistics, spectral moment ratios, spectral band strengths, and local binary model based statistics from multi-channel EMG data were classified by k-NN and probabilistic neural networks. In experimental studies, they achieved an accuracy rate of $92.7 \%$ with probabilistic neural networks and 93.9\% k-NN. Alçin [11] extracted features from EEG signals using Fractal Detrended Fluctuation Analysis (F-DFA) and non-overlapping window Root Mean Square (w-RMS) methods. These features are classified using methods such as SVM, k-NN, LDA and decision trees. In the experimental studies, they achieved $96.83 \%$ success.

In this study, it is aimed to define some basic hand gestures using the three-channel sEMG data set. In the proposed method, Dispersion Entropy (DisEn) and Normal Cumulative Distribution Function (NCDF), which have never been used in feature extraction from EMG signals before, are used for feature extraction. Using linear mapping in feature extraction with DisEn in time series, sometimes the maximum/minimum values of the time series might be smaller or larger than the mean/median values of the signal. This might cause the signal to be assigned only a few classes. To eliminate this situation, NCDF is used in feature extraction. An increase is observed in classification performance by combining DisEn and NCDF features of each channel. Comparing the performance of SVM and ELM methods, which are commonly preferred for classification in the proposed method, higher success was obtained with SVM.

The rest of this work is organized as follows. Dataset, Feature extraction, and classification methods are described in the second chapter. In the third chapter, experimental studies and results are explained in detail. In the last part, the results of the proposed study are discussed.

\section{Material and Method}

In this section, the methods used for the data set, feature extraction, and classification are described in detail.

\subsection{Data Set}

The EMG data set includes movements of five healthy participants, three of which are female, to hold and grasp different objects with the surface electrodes attached to the forearm. Participants performed 6 
different hand gestures, including holding a heavy load (hook), holding an object facing the palm (palm), holding a thin flat small object (lat), holding a spherical object (spher), holding a small object with the fingertips (tip), and holding a cylindrical object (cyl). For each movement, 100 measurements with three channels were recorded and each channel contains 2500 samples. Figure 1 shows the six hand movements of a participant and the signal of each channel of these movements. Also, the data set consists of 600 signals in total, and a $15-500 \mathrm{~Hz}$ bandpass filter and $50 \mathrm{~Hz}$ notch noise canceling filter are used for each signal [5].

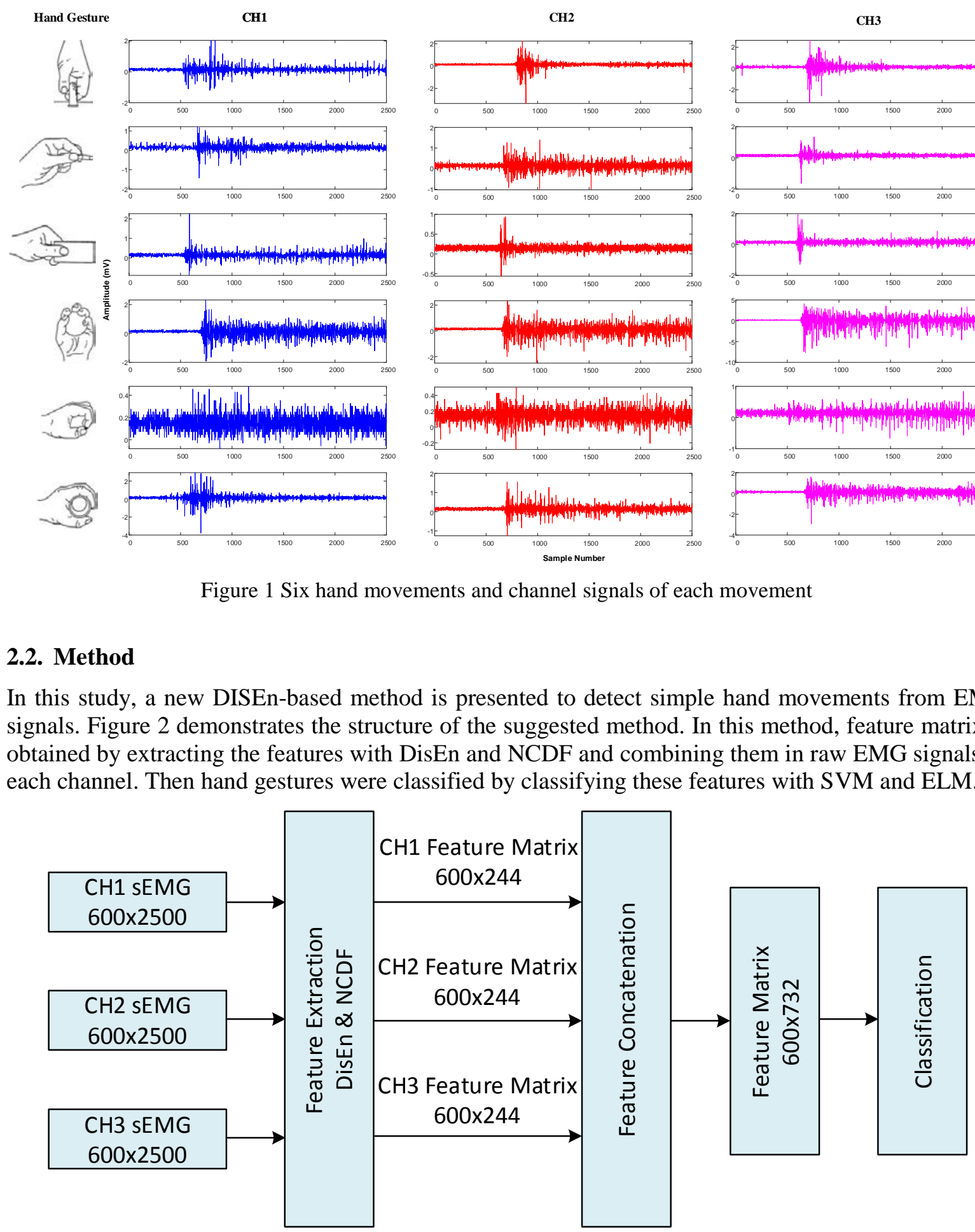

Figure 2 Structure of suggested method 


\subsubsection{Dispersion Entropy}

Entropy is one of the substantial methods preferred to evaluate the dynamic properties of time series. Entropy was introduced by Shannon in 1948 to describe a measure of uncertainty or disorder in data theory [12]. This concept can also be defined as the regularity quantification of a system or time series using the probability distribution of situations. Although there are many entropy methods, those such as Permutation Entropy (PEn) and Sample Entropy (SEn) are widely used to measure the irregularity of signals on a temporal scale [13]. Thus, although both methods have widespread use in biomedical signal and image processing applications, SEn is not fast enough for some real-time applications and longterm signals. In addition, the fact that PEn does not take the differences between the average value of the signal amplitudes and the amplitude values into account stands out as the most important limitations of these methods [14]. Recently, the DisEn method has been recommended to overcome the limitations of these methods. The DisEn method is used more in biomedical applications since it is also sensitive to frequency changes and the calculation time is very short [15].

Suppose we have an $\mathrm{N}$-dimensional time series with a single variable as shown $x_{i}=\left\{x_{1}, x_{2}, \ldots, x_{N}\right\}$. The DisEn algorithm follows the following processes for the analysis of this signal [15] [16];

a. It first matches the time series with the mapping function that calculates and uses mean and standard deviation values of the time series.

b. Class numbers (nc) are matched to the signal obtained by distributing it along the amplitude range. Each sample is transferred to the nearest relevant class depending on the amplitude. Linear and nonlinear mapping approaches are used for this processing. Although linear mapping algorithms are fast, the maximum / minimum values of the time series can sometimes be much smaller or larger than the mean / median values of the signal. In this case only a few classes of signal are assigned to any class. This causes a lot of data to be assigned to any class. To solve this problem, first the time series $x_{i}$ NCDF is used to obtain a signal $y_{j}=\left\{y_{1}, y_{2}, \ldots, y_{N}\right\}$ with a value between 0 and 1 . Then, each $y_{j}$ is removed by assigned to an integer from one to nc, which is the class number, with the linear mapping algorithm.

c. Based on embedding size (m) and time delay (d) $y_{i}^{m, n c}=\left\{y_{i}^{n c}, y_{i+d}^{n c},+\cdots+y_{i+(m-1) d}^{n c}\right\}$ and $i=1,2, \ldots, N-(m-1) d$, each time series is matched to its distribution model. The number of potential distribution patterns is $y_{i}^{m, n c}$.

d. The relative frequencies for the potential distribution models for each $\mathrm{nc}^{\mathrm{m}}$ are obtained and the DisEn value of the time series is calculated with the help of Eq. (1) depending on the Shannon definition of entropy.

$$
\operatorname{DisEn}(x, m, n c, d)=\sum_{\pi=1}^{n c^{m}} p\left(\pi_{v_{0} v_{1} \ldots v_{m-1}}\right) \cdot \ln \left(p\left(\pi_{v_{0} v_{1} \ldots v_{m-1}}\right)\right)
$$

Here $p\left(\pi_{v_{0} v_{1} \ldots v_{m-1}}\right)$ shows the total number of distribution models. For detailed information about the method [12], [15] can be examined. In this study, NCDF obtained while calculating DisEnt is thought to have a distinctive feature. Thus, $n c^{m}$ number of features are obtained from one EMG signal.

\subsubsection{Support Vector Machine}

SVM is a supervised learning algorithm frequently preferred for linear and nonlinear data classification [17]. Its basic structure is to find the best hyperplane that will provide the maximum margin among the output classes. Suppose the data set is $D=\left\{\left(x_{1}, y_{1}\right),\left(x_{2}, y_{2}\right), \ldots,\left(x_{n}, y_{n}\right)\right\}$. Here, $x_{n} \in R$ defines the number of elements of the data set $\mathrm{n}$ and $y_{n} \in\{-1,1\}$ defines the class labels of $x_{n}$ [18]. Accordingly, the decision function that will find the best hyperplane can be written as in Eq. (2).

$$
y_{n}\left(w^{T} x_{n}+b\right) \geq 1
$$


Here $\mathrm{w}$ defines $\mathrm{n}$-dimensional weights and $\mathrm{b}$ defines the threshold value used to determine the hyperplane position. However, in order to find the best hyperplane with the decision function in Eq. (2), the maximum distance calculated with $d=\max \left(\frac{1}{\|w\|}\right)$ should be minimized $\min \frac{\|w\|^{2}}{2}$ [19]. Also, equation 2 can be valid if the given and predicted answers have the same sign. The fact that given and predicted answers have different signs, which is a significant problem for SVM, can be solved with Lagrange optimization given in Eq. (3) [20].

$$
L(\alpha)=\sum_{n=1}^{N} \alpha_{n}-\frac{1}{2} \sum_{n, j=1}^{N} \alpha_{n} \alpha_{j} y_{n} y_{j} k\left(y_{n}, y_{j}\right)
$$

Here, $k\left(y_{n}, y_{j}\right)$ shows kernel function and $\alpha_{i}$ shows Lagrange multiplier.

\subsubsection{Extreme learning Machine}

ELM is a learning algorithm for single-hidden layer feedforward networks [21], [22]. In ELM, input weights and hidden layer threshold values are started randomly and maintained stable. In addition, Since the output weights are calculated analytically, it has a fast learning process. ELM calculates the output weights with a simple linear equation. Output weights can be found by Eq. (4).

$$
Y=H \cdot \beta
$$

Here, $\mathrm{Y}$ is real output value, $\mathrm{H}$ is matrices of weighted inputs includes activation function step and $\beta$ is the desired output weights vector [21, 22]. ELM is commonly used as it exhibits better generalization performance compared to conventional feedforward networks. For more detailed information about the ELM method, see [22].

\section{Experimental Study and Results}

In the study, NCDF approach, which was not used in feature extraction from EMG signals before, and DisEn were used in feature extraction. Experimental studies were tested with a 3 channel EMG dataset, consisting of 600 records and containing six basic hand movements. In the suggested method, DisEn and NCDF parameters are calculated as distinctive features. Motion estimation was made by applying these features to SVM and ELM classifiers. 10-fold cross-validation was used to demonstrate the validity of the method in all experimental studies. The experimental study was conducted in three steps. First, with DisEn only, a total of $600 \times 3$ feature matrices were calculated, $600 \times 1$ for each channel. The classification accuracy of hand movements depending on these features and $m$ and $n c$ values can be seen in Figure. 3.

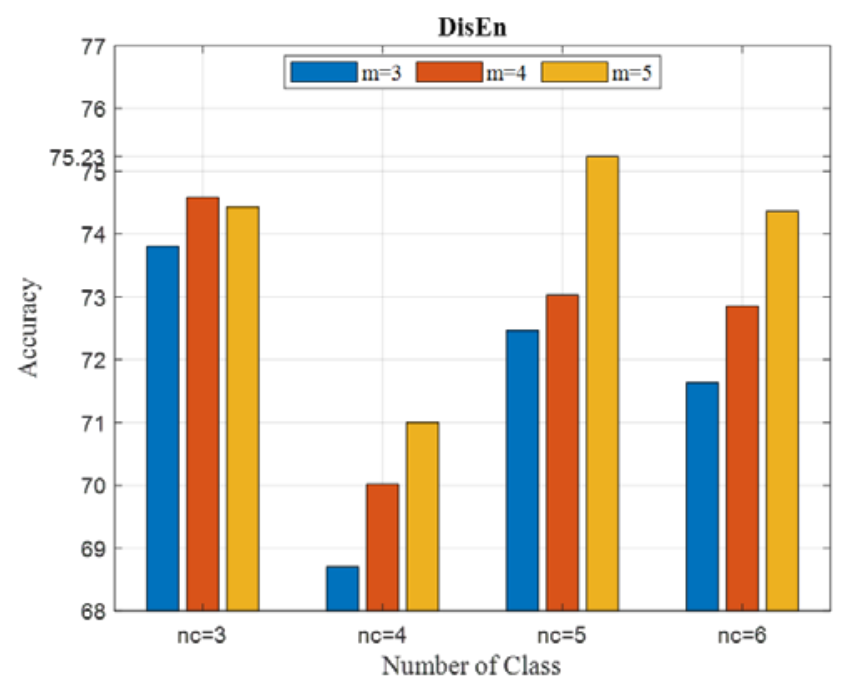

Figure 3 Hand gestures recognition achievements of DisEn features according to the number of classes 
As seen in Figure 3, the highest performance was obtained as $75.23 \%$ for $\mathrm{m}=5$ and $\mathrm{nc}=5$ values. The closest values to this success were obtained for $n c=3, m=4$ and $n c=6, m=5$ values. In the second phase, motion estimation has been made for each channel with NCDF features. At this stage, the classification performance of hand movements depending on the $m$ and $n c$ values is shown in Figure 4. As seen here, the highest performance achieved was $97.17 \%$ for $m=5$ and $n c=3$ values. In the first two stages, the results of the SVM classifier are given due to its performance. It is also seen that NCDF features are more distinguishing for EMG signals compared to the DisEn method. By using the nc and $m$ values that provide the highest performance with experimental studies, $\left(n \mathrm{c}^{\mathrm{m}}=3^{5}\right) 243$ features are obtained for each channel.

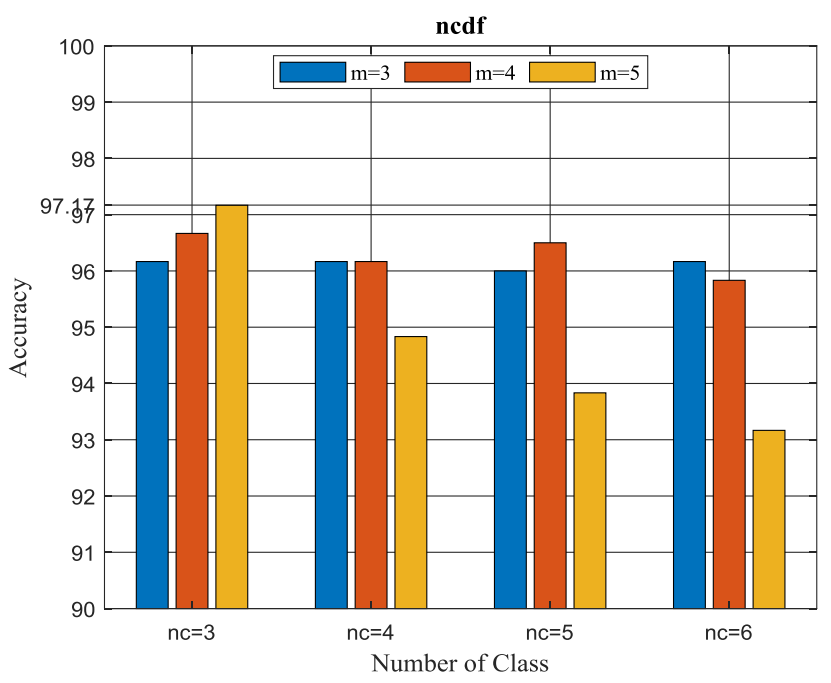

Figure 4 Hand gestures recognition performance of NCDF features according to the number of classes.

In the last experimental stage, a $600 \times 244$ feature matrix was generated by combining the features of each channel $(600 \times 1$ with DisEn and $600 \times 243$ with NCDF) in two previous experimental studies. A total of $600 \times 732$ feature matrix is obtained for three channels. In the third stage, confusion matrix and the highest performance were taken into account, and $n c=3$ and $m=5$ values were used. Also, the individual and combined features are evaluated with the ELM classifier in this stage. The individual and combined performance results of the calculated features are given in Table 1.

Table 1 Hand gestures recognition performance of NCDF features according to the number of classes.

\begin{tabular}{lcc}
\hline \multirow{2}{*}{ Method } & \multicolumn{2}{c}{ Accuracy (\%) } \\
\cline { 2 - 3 } & SVM & ELM \\
\hline DisEn & 75.1667 & 57.3333 \\
\hline NCDF & 97.1700 & 95.3333 \\
\hline DisEn+NCDF & $\mathbf{9 8 . 0 0 0 0}$ & 96.6667 \\
\hline
\end{tabular}

As shown in Table 2, the SVM classifier produced better results compared to ELM. The combination of DisEn and NCDF features from Table 1 made positive contributions to overall performance. The confusion matrix of DisEn + NCDF and SVM model because of the highest performance is given in Figure 5.

As seen in Figure 5, the lowest performance with $93 \%$ was obtained in the action of holding the palm facing the object (palmar). While 99\% performance was achieved in holding small objects and holding cylindrical objects with fingertips, the highest performance was achieved as $100 \%$ in heavy load holding and spherical object holding actions. Seven of the actions of holding the palm with the palm facing the object with the lowest performance were incorrectly classified as holding a flat object. In addition, the performance of the suggested method and comparative results of the methods using the same data set are given in Table 2. 


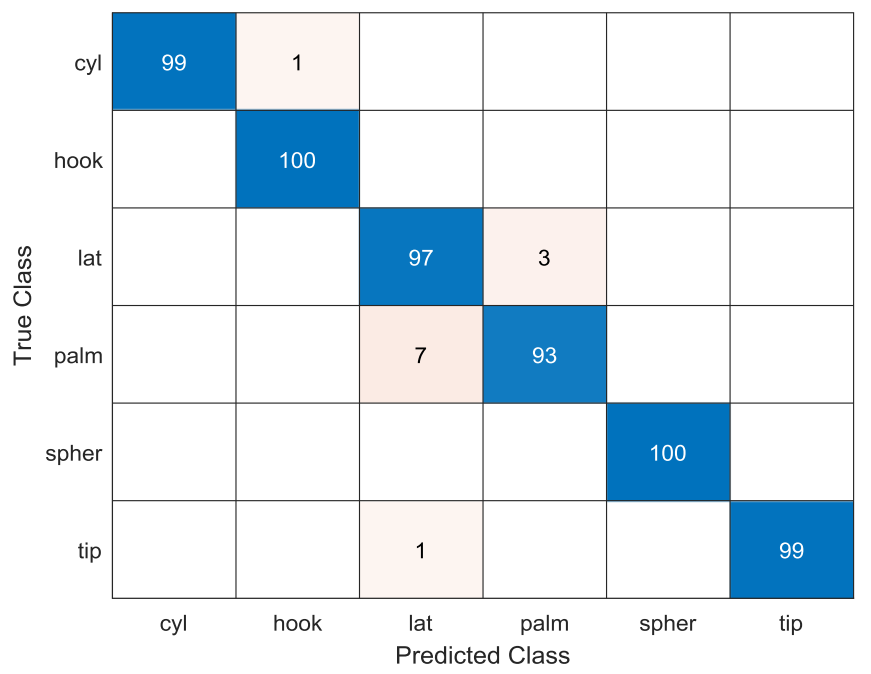

Figure 5 Proposed method confusion matrix

When Table 2 is examined it is seen that there is a performance between $80 \%$ and $96.833 \%$ for the EMG data set. The suggested method has approximately $1.16 \%$ better accuracy than the method with the highest success in the literature. The comparison results show that the proposed method can be used effectively in classifying EMG signals.

Table 2. Comparison of the proposed method to the methods using the same data set

\begin{tabular}{ll}
\hline Method & Accuracy (\%) \\
\hline Sapsanis et. al. [5] & 80.000 \\
\hline Ar1 et. al. [4] & 90.000 \\
\hline Ar1 et. al. [8] & 93.167 \\
\hline Alçin O.F. [11] & 96.833 \\
\hline Suggested Method & $\mathbf{9 8 . 0 0 0}$ \\
\hline
\end{tabular}

\section{Discussion}

In this study, a new method based on feature extraction by NCDF, which has never been used in feature extraction from EMG signals before, is suggested. In order to increase the performance of the DisEn method, which is frequently used in obtaining the distinctive properties of biomedical signals, its suitability for the classification of EMG signals in combining them with NCDF features has been studied. In the studies conducted with the data set involving six basic hand gestures, approximately $22 \%$ better classification performance was achieved in the classification of NCDF features compared to the classification of DisEn features. When features of both methods are combined, hand movements were detected with 98\% success. Compared to existing methods with the same data set, the method has approximately $1.16 \%$ higher success than the best study available in the literature. Considering the results of the study, it shows the usability of the suggested method in biomedical rehabilitation, prosthesis, and robotics applications. As a future study, we could focus to implement the proposed method in embedded devices.

\section{References}

[1] J. J. A. Mendes Junior, M. L. B. Freitas, H. V. Siqueira, A. E. Lazzaretti, S. F. Pichorim, and S. L. Stevan, "Feature selection and dimensionality reduction: An extensive comparison in hand gesture classification by sEMG in eight channels armband approach," Biomed. Signal Process. 
Control, vol. 59, 2020, doi: 10.1016/j.bspc.2020.101920.

[2] Babita, P. Kumari, Y. Narayan, and L. Mathew, "Binary movement classification of sEMG signal using linear SVM and Wavelet Packet Transform," 1st IEEE Int. Conf. Power Electron. Intell. Control Energy Syst. ICPEICES 2016, pp. 30-33, 2017, doi: 10.1109/ICPEICES.2016.7853640.

[3] C. Sravani, V. Bajaj, S. Taran, and A. Sengur, "Flexible Analytic Wavelet Transform Based Features for Physical Action Identification Using sEMG Signals," IRBM, 2020, doi: 10.1016/j.irbm.2019.07.002.

[4] A. Arı, F. AYAZ, and D. HANBAY, "EMG Sinyallerinin Kısa Zamanlı Fourier Dönüşüm Özellikleri Kullanılarak Yapay Sinir Ağları ile Sınıflandırılması," Fırat Üniversitesi Mühendislik Bilim. Derg., pp. 443-451, Sep. 2019, doi: 10.35234/fumbd.545161.

[5] C. Sapsanis, G. Georgoulas, A. Tzes, and D. Lymberopoulos, "Improving EMG based classification of basic hand movements using EMD," Proc. Annu. Int. Conf. IEEE Eng. Med. Biol. Soc. EMBS, pp. 5754-5757, 2013, doi: 10.1109/EMBC.2013.6610858.

[6] J. Qi, G. Jiang, G. Li, Y. Sun, and B. Tao, "Intelligent Human-Computer Interaction Based on Surface EMG Gesture Recognition," IEEE Access, vol. 7, pp. 61378-61387, 2019, doi: 10.1109/ACCESS.2019.2914728.

[7] N. A. Chaya, B. R. Bhavana, S. B. Anoogna, M. Hiranmai, and N. B. Krupa, "Real-Time Replication of Arm Movements Using Surface EMG Signals," Procedia Comput. Sci., vol. 154, pp. 186-193, 2018, doi: 10.1016/j.procs.2019.06.028.

[8] A. Arı, B. Arı, and Ö. F. Alçin, "Elektromiyografi Sinyallerinin Permütasyon Entropi ve Bir Boyutlu Yerel İkili Özellikler Kullanılarak Sınıflandırılması," J. Tepecik Educ. Res. Hosp., vol. 30, no. 1, pp. 46-49, 2020, [Online]. Available:

https://www.journalagent.com/terh/pdfs/TERH_30_1_1_82.pdf.

[9] T. Tuncer, S. Dogan, and A. Subasi, "Surface EMG signal classification using ternary pattern and discrete wavelet transform based feature extraction for hand movement recognition," Biomed. Signal Process. Control, vol. 58, p. 101872, 2020, doi: 10.1016/j.bspc.2020.101872.

[10] A. C. Turlapaty and B. Gokaraju, "Feature Analysis for Classification of Physical Actions Using Surface EMG Data," IEEE Sens. J., vol. 19, no. 24, pp. 12196-12204, 2019, doi: 10.1109/JSEN.2019.2937979.

[11] Ö. F. Alçin, "Fraktal Eğimden Arındırılmış Dalgalılık Analizi ve Pencereli Kare Ortalamanın Karekökü Tabanlı EMG Sınıflandırma," Fırat Üniversitesi Mühendislik Bilim. Derg., vol. 32, no. 2, pp. 359-368, 2020, doi: 10.35234/fumbd.771205.

[12] M. Rostaghi and H. Azami, "Dispersion Entropy: A Measure for Time-Series Analysis," IEEE Signal Process. Lett., vol. 23, no. 5, pp. 610-614, 2016, doi: 10.1109/LSP.2016.2542881.

[13] H. Azami et al., "Multiscale fluctuation-based dispersion entropy and its applications to neurological diseases," IEEE Access, vol. 7, pp. 68718-68733, 2019, doi: 
10.1109/ACCESS.2019.2918560.

[14] M. Zanin, L. Zunino, O. A. Rosso, and D. Papo, "Permutation entropy and its main biomedical and econophysics applications: A review," Entropy, vol. 14, no. 8, pp. 1553-1577, 2012, doi: $10.3390 / \mathrm{e} 14081553$.

[15] H. Azami, L. E. V. da Silva, A. C. M. Omoto, and A. Humeau-Heurtier, "Two-dimensional dispersion entropy: An information-theoretic method for irregularity analysis of images," Signal Process. Image Commun., vol. 75, no. April, pp. 178-187, 2019, doi: 10.1016/j.image.2019.04.013.

[16] E. Kafantaris, I. Piper, T. Y. M. Lo, and J. Escudero, "Augmentation of dispersion entropy for handling missing and outlier samples in physiological signal monitoring," Entropy, vol. 22, no. 3, 2020, doi: 10.3390/e22030319.

[17] M. Aslan, Y. Akbulut, A. Şengür, and M. C. Ince, "Skeleton based efficient fall detection," J. Fac. Eng. Archit. Gazi Univ., 2017, doi: 10.17341/gazimmfd.369347.

[18] F. Demir, M. Turkoglu, M. Aslan, and A. Sengur, "A new pyramidal concatenated CNN approach for environmental sound classification," Appl. Acoust., 2020, doi: 10.1016/j.apacoust.2020.107520.

[19] S. Yu, X. Li, X. Zhang, and H. Wang, "The OCS-SVM: An Objective-Cost-Sensitive SVM With Sample-Based Misclassification Cost Invariance," IEEE Access, vol. 7, pp. 118931118942, 2019, doi: 10.1109/access.2019.2933437.

[20] X. Wu, W. Zuo, L. Lin, W. Jia, and D. Zhang, "F-SVM: Combination of Feature Transformation and SVM Learning via Convex Relaxation," IEEE Trans. Neural Networks Learn. Syst., vol. 29, no. 11, pp. 5185-5199, 2018, doi: 10.1109/TNNLS.2018.2791507.

[21] O. F. Alcin, A. Sengur, J. Qian, and M. C. Ince, "OMP-ELM: Orthogonal matching pursuitbased extreme learning machine for regression," J. Intell. Syst., 2015, doi: 10.1515/jisys-20140095.

[22] G. Bin Huang, Q. Y. Zhu, and C. K. Siew, "Extreme learning machine: Theory and applications," Neurocomputing, 2006, doi: 10.1016/j.neucom.2005.12.126. 